Paper

\title{
An approximation for dynamical processes on periodic temporal networks
}

\author{
Naoya Fujiwara ${ }^{1 a)}$ \\ ${ }^{1}$ Center for Spatial Information Science, The University of Tokyo \\ 5-1-5 Kashiwanoha, Kashiwa-shi, Chiba 277-8568, Japan \\ a)fujiwara@csis.u-tokyo.ac.jp
}

Received September 18, 2014; Revised November 22, 2014; Published April 1, 2015

\begin{abstract}
Many dynamical processes such as the spread of epidemic diseases take place on temporal networks. In this paper, we study a special but important case of the temporal networks where the network topology changes periodically in time. We derive the time evolution operator based on the Floquet theory by expanding the periodic Laplacian matrix with respect to the coupling strength. In particular, the first and the second order terms of the expansion are explicitly given with respect to the time integral of the product of the time dependent Laplacian matrices. Using this series expansion, response of the system to a perturbation is also presented. In particular, the deviation from the fast switching approximation, which replaces the time dependent Laplacian matrix with its time average, appears in the lowest order term of the expansion.
\end{abstract}

Key Words: temporal networks, Floquet theory, dynamical processes on networks

\section{Introduction}

Temporal network $[1,2]$ is an important subclass of complex networks, where the network topology is time-dependent hence the connectivity matrix or the Laplacian matrix characterizing the network topology is not static. Heavy tail and predictability of temporal networks has been studied [3]. It is worth noting that the time dependence of a lot of interesting temporal networks is periodic. The periodicity of such periodic social and natural networks is caused by the daily, weekly, seasonal changes of the Earth system.

Dynamical processes and nonlinear dynamics on complex networks $[4,5]$ can be considered as an extension of dynamics on lower dimensional systems, e.g. spread of infectious diseases [6], synchronization phenomena [7,8], social systems [9], etc. Recently, further extension of those dynamical processes to the temporal networks has been considered in epidemics [10], biological systems [11], and social balance problem [12].

The Laplacian matrices often appear in the dynamics on complex networks, particularly in synchronization phenomena on temporal networks. In order to understand synchronization, an approximation of the fast switching topology has been intensively applied [13-16]. This approximation replaces the time-dependent Laplacian matrix with its time average, assuming that the time scale of topological change is much faster than that of the dynamics. However, the deviation from this simplification has 
been observed if this assumption does not hold [17-19].

How does this deviation from the fast switching approximation arise? It is necessary to answer this question from the network structure and time evolution of the network. As we will see later in this paper, periodic average of the Laplacian matrix describes the long-term behavior of the system. One example where the time dependence of the Laplacian is dominant is when an external forcing is put on the system. In such a case, the effect of the forcing depends on the phase of the periodic change of the temporal network.

From engineering point of view, an external forcing is applied in order to control the dynamics on the temporal networks. Continuous time translational symmetry breaks in the temporal networks, and the response of the system to the perturbation depends on the phase of the network. This dependence of the response on the phase cannot be captured by the fast switching approximation. Synchronizability of coupled nonlinear oscillator network systems also has been studied using the Lyapunov function, and some criteria for synchronization and control protocols to achieve synchronization have been proposed [20-22]. However, these criteria are often sufficient condition which can be too conservative. Even if the algorithm to stabilize the system is known with the Lyapunov function, it may be computationally complex. For example, although a switching rule to stabilize a system exists, the algorithm to yield this rule can be NP-hard [23,24]. If one is interested in the dynamics on complex networks from scientific viewpoint, another analytical method to see the details of dynamical behavior should be developed. Furthermore, such a detailed analysis may lead us to a better criterion for synchronization.

As mentioned above, there are many realistic systems where the network topology changes periodically in time. Here we study a linear dynamical system described by the time periodic Laplacian matrix as an example, and propose a methodology specific to the periodic systems. The Floquet theory, which gives the averaged time evolution over one period, is suitable for this purpose. Although the Floquet theory is powerful, we have to take into account the size of giant complex networks: it is not realistic to compute the Floquet matrix, because it requires to compute the product of the time dependent Laplacian matrices. In order to solve this problem, we propose an approximation employing the series expansion of the monodromy matrix with respect to the coupling strength. Using this expansion, we describe the system's response to the perturbation over one period. This approach can shed light on the dynamical processes on temporal networks which are periodic.

This paper is organized as follows. In Sec. 2, the formalism of the power series expansion of the Floquet matrix of the periodic Laplacian matrix is given. Series expansion of the response to an external forcing, which enables us to discuss the effect of the forcing, is considered in Sec. 3. This formalism is applied in a simple example and it is shown how this approximation works in Sec. 4 . The results are summarized and discussed in the last section.

\section{Series expansion of the Floquet matrix}

In the present study, we consider a simplest case of the dynamical processes on a periodic temporal network described by the time-dependent Laplacian matrix $L(t)$ as

$$
\dot{x}=-\beta L(t) x,
$$

where each component $x \in \mathbb{R}^{n}$ corresponds to the dynamical variable on a node, and the periodicity $L(t+T)=L(t)$ holds. Matrix elements $L_{i j}(t)=L_{j i}(t)=-1$ if nodes $i$ and $j$ are connected at time $t$, and $L_{i i}(t)=k_{i}(t)$, where $k_{i}(t)$ denotes the instantaneous degree of the node $i$. Sum of each row of the Laplacian matrix is zero, and its smallest eigenvalue is zero. The coupling strength $\beta$ determines the time scale of the dynamics. This simple but important model is used to describe opinion formation, synchronization phenomena, etc.

The solution of this equation is

$$
\begin{aligned}
x(t) & =U(t ; 0) x(0) \\
& =\mathcal{T} \exp \left[-\beta \int_{0}^{t} d t_{1} L\left(t_{1}\right)\right] x(0),
\end{aligned}
$$


where $U(t ; 0)$ is the time evolution operator from $t=0$ to $t$, and $\mathcal{T}$ denotes the time ordered operation. This solution can be expanded in terms of $\beta$, and the expansion up to the second order is

$$
U(t ; 0)=1-\beta \int_{0}^{t} d t_{1} L\left(t_{1}\right)+\beta^{2} \int_{0}^{t} d t_{1} \int_{0}^{t_{1}} d t_{2} L\left(t_{1}\right) L\left(t_{2}\right)+O\left(\beta^{3}\right) .
$$

Note the equality of the time ordered product $\int_{0}^{t} d t_{1} \int_{0}^{t} d t_{2} \mathcal{T}\left[L\left(t_{1}\right) L\left(t_{2}\right)\right]=2 \int_{0}^{t} d t_{1} \int_{0}^{t_{1}} d t_{2} L\left(t_{1}\right) L\left(t_{2}\right)$ holds. Since $L(t)$ is periodic, the decomposition

$$
U(t ; 0)=S(t) e^{-t \Lambda}
$$

is possible according to the Floquet theorem. Here, the periodic matrix $S(t+T)=S(t)$ satisfies $S(0)=S(T)=I_{n}$, where $I_{n}$ denotes the identity matrix of size $n$. Furthermore, the constant matrix $\Lambda$ characterizes the long-term exponential decay of the dynamics. Since $S(T)=I_{n}$ holds, we obtain

$$
\begin{aligned}
e^{-T \Lambda} & =U(T ; 0) \\
& =\left(1-\beta T \Lambda_{1}+\beta^{2} T^{2} \Lambda_{2}\right)+O\left(\beta^{3}\right),
\end{aligned}
$$

and

$$
e^{T \Lambda}=1+\beta T \Lambda_{1}+\beta^{2} T^{2}\left(\Lambda_{1}^{2}-\Lambda_{2}\right)+O\left(\beta^{3}\right),
$$

where

$$
\begin{aligned}
\Lambda_{1} & =\frac{1}{T} \int_{0}^{T} d t_{1} L\left(t_{1}\right), \\
\Lambda_{2} & =\frac{1}{T^{2}} \int_{0}^{T} d t_{1} \int_{0}^{t_{1}} d t_{2} L\left(t_{1}\right) L\left(t_{2}\right) .
\end{aligned}
$$

Note that Eq. (8) can be derived from $e^{T \Lambda} e^{-T \Lambda}=I_{n}$ and Eq. (7).

Our goal is to obtain the series expansion of $S(t)$ with respect to $\beta$. The exponential part $e^{t \Lambda}$ can be expanded as

$$
e^{t \Lambda}=1+\beta t \Lambda_{1}+\beta^{2} t^{2}\left(\Lambda_{1}^{2}-\Lambda_{2}\right)+O\left(\beta^{3}\right) .
$$

Then, it is possible to derive $S(t)$ from Eqs. (5), (4), and (11) as

$$
\begin{aligned}
S(t)= & U(t ; 0) e^{t \Lambda} \\
= & 1+\beta\left[t \Lambda_{1}-\int_{0}^{t} d t_{1} L\left(t_{1}\right)\right]+\beta^{2}\left\{t^{2}\left(\Lambda_{1}^{2}-\Lambda_{2}\right)-t \int_{0}^{t} d t_{1} L\left(t_{1}\right) \Lambda_{1}\right. \\
& \left.+\int_{0}^{t} d t_{1} \int_{0}^{t_{1}} d t_{2} L\left(t_{1}\right) L\left(t_{2}\right)\right\}+O\left(\beta^{3}\right) .
\end{aligned}
$$

We can easily verify that $S(0)=1$ and $S(T)=1+O\left(\beta^{3}\right)$ hold by substituting $t=T$ to the above equation, hence the periodicity of $S$ is guaranteed up to $O\left(\beta^{2}\right)$ with this expansion as expected.

The inverse of $S(t)$ can be derived from $S^{-1}(t) S(t)=I_{n}$ as

$$
\begin{aligned}
S^{-1}(t)= & 1-\beta\left[t \Lambda_{1}-\int_{0}^{t} d t_{1} L\left(t_{1}\right)\right]+\beta^{2}\left\{t^{2} \Lambda_{2}-t \int_{0}^{t} d t_{1} \Lambda_{1} L\left(t_{1}\right)\right. \\
& \left.+\int_{0}^{t_{2}} d t_{1} \int_{0}^{t} d t_{2} L\left(t_{1}\right) L\left(t_{2}\right)\right\}+O\left(\beta^{3}\right) .
\end{aligned}
$$

Note that the fast switching approximation assumes $S(t)=I_{n}$. The $O(\beta)$ terms of $S(t)$ represents the lowest order correction from this approximation. 


\section{Response to perturbations of periodic temporal networks}

In this section, we discuss the response to the external forcing using the series expansion. We consider the evolution equation with the external forcing $p(t)$ as

$$
\dot{x}(t)=-\beta L(t) x(t)+p(t) .
$$

This equation can be integrated over one period as

$$
x((m+1) T)=e^{-T \Lambda} x(m T)+\int_{0}^{T} d t_{1} e^{-\left(T-t_{1}\right) \Lambda} S^{-1}\left(t_{1}\right) p\left(m T+t_{1}\right) .
$$

Here, $m$ is an integer.

In general, the response of the system can be understood if we can compute $S(t)$. However, it requires to compute the product of the Laplacian matrices, which is computationally heavy. Therefore, it is important to derive the series expansion of $e^{-\Lambda\left(T-t_{1}\right)} S^{-1}\left(t_{1}\right)$ with respect to $\beta$, which enables us to approximate it with the sum or product of a few Laplacian matrices. We cannot estimate the dependence of the response of $x$ on the phase of the Laplacian matrix under the fast switching approximation, which corresponds to taking $S(t)=I_{n}$ and replacing $L(t)$ in Eq. (15) with its periodic average $\Lambda_{1}$. The integrand in Eq. (16) follows from the expansions Eqs. (7) and (14) as

$$
e^{-\left(T-t_{1}\right) \Lambda} S^{-1}\left(t_{1}\right) p\left(m T+t_{1}\right)=\left\{1-\beta\left[T \Lambda_{1}-\int_{0}^{t_{1}} d t_{2} L\left(t_{2}\right)\right]\right\} p\left(m T+t_{1}\right)+O\left(\beta^{2}\right) .
$$

With the fast switching approximation, the $O(\beta)$ term is $\beta\left(T-t_{1}\right) \Lambda_{1} p\left(m T+t_{1}\right)$. The lowest order correction of the response to the forcing is the deviation of the time integral of the Laplacian matrix, $\int_{0}^{t_{1}} d t_{2} L\left(t_{2}\right)$, from the periodic average $t_{1} \Lambda_{1}$.

Finally we briefly mention the spectral decomposition of this equation. This procedure has been introduced in the phase reduction [8], where Eq. (17) is decomposed into the eigenvectors of $\Lambda$. We denote the $a$ th eigenvalue of $\Lambda$ as $\lambda_{a}$, and the corresponding left and right eigenvectors as $v_{a}^{*}$ and $v_{a}$. The left eigenvector $v_{a}^{*}$ is normalized as $v_{a}^{*} v_{b}=\delta_{a b}$, where $\delta_{a b}$ denotes the Kronecker's delta. Then one can decompose $x(t)$ and the forcing term as

$$
\begin{aligned}
x(t) & =\sum_{a} X_{a}(t) v_{a}, \\
S^{-1}(t) p(t) & =\sum_{a} P_{a}(t) v_{a} .
\end{aligned}
$$

Note that the coefficients can be obtained with $X_{a}(t)=v_{a}^{*} x(t)$ and $P_{a}(t)=v_{a}^{*} S^{-1}(t) p(t)$. Substituting these expansions to Eq. (16), we obtain

$$
X_{a}((m+1) T)=e^{-T \lambda_{a}} X_{a}(m T)+\int_{0}^{T} d t_{1} e^{-\left(T-t_{1}\right) \lambda_{a}} P_{a}\left(m T+t_{1}\right)
$$

However, it is in general difficult to compute $\Lambda$, hence $\lambda_{a}, v_{a}$, and $v_{b}^{*}$. Instead, if we apply Eq. (17) and use the eigenvalue $\lambda_{a}^{(1)}$ and right and left eigenvector, $v_{a}^{(1)}$ and $v_{a}^{(1) *}$ of $\Lambda_{1}$, the equation similar to Eq. (20) can be achieved as

$$
\begin{aligned}
X_{a}((m+1) T)= & \left(1-\beta T \lambda_{a}^{(1)}\right) X_{a}(m T) \\
& +\int_{0}^{T} d t_{1}\left\{1-\beta\left[T \lambda_{a}^{(1)}-\beta \int_{0}^{t_{1}} d t_{2} v_{a}^{(1) *} L\left(t_{2}\right) v_{a}^{(1)}\right]\right\} P_{a}\left(m T+t_{1}\right) \\
& +O\left(\beta^{2}\right) .
\end{aligned}
$$

If an eigenvalue $\lambda_{a}$, which represent the linear growth rate of the $a$ th mode, is so small that associated $X_{a}(t)$ is much smaller than $X_{1}(t)$, one can neglect $X_{a}$. Then the dynamics can be reduced to the equation for a few dominant modes. 


\section{Example}

In this section, we see how this approximation works using a simple example. Let us consider a network with three nodes and connections alter between two patterns every $\tau$ (Fig. 1). Hence the period of this network is $T=2 \tau$. We consider the evolution Eq. (1) with $L(t)=L_{2}$ for $m T \leq t \leq m T+\tau$ and $L(t)=L_{3}$ for $m T+\tau \leq t \leq(m+1) T$, where the Laplacian matrices of the two patterns are

$$
L_{2}=\left(\begin{array}{ccc}
1 & -1 & 0 \\
-1 & 2 & -1 \\
0 & -1 & 1
\end{array}\right), L_{3}=\left(\begin{array}{ccc}
1 & 0 & -1 \\
0 & 1 & -1 \\
-1 & -1 & 2
\end{array}\right)
$$

The time evolution operator is

$$
U(t ; 0)= \begin{cases}e^{-\beta t L_{2}} & (0 \leq t \leq \tau), \\ e^{-\beta(t-\tau) L_{3}} e^{-\beta \tau L_{2}} & (\tau \leq t \leq T),\end{cases}
$$

and particularly

$$
\begin{aligned}
U(T ; 0) & =e^{-2 \tau \Lambda} \\
& =e^{-\beta \tau L_{3}} e^{-\beta \tau L_{2}}
\end{aligned}
$$

holds. This implies

$$
e^{-t \Lambda}=e^{-\beta t L_{3} / 2} e^{-\beta t L_{2} / 2}
$$

and

$$
e^{t \Lambda}=e^{\beta t L_{2} / 2} e^{\beta t L_{3} / 2}
$$

Using Eq. (5), we have

$$
\begin{aligned}
S(t) & = \begin{cases}e^{-\beta t L_{2} / 2} e^{\beta t L_{3} / 2} & (0 \leq t \leq \tau), \\
e^{-\beta(t-\tau) L_{3}} e^{-\beta(\tau-t / 2) L_{2}} e^{\beta t L_{3} / 2} & (\tau \leq t \leq T),\end{cases} \\
S^{-1}(t) & = \begin{cases}e^{-\beta t L_{3} / 2} e^{\beta t L_{2} / 2} & (0 \leq t \leq \tau), \\
e^{-\beta t L_{3} / 2} e^{\beta(\tau-t / 2) L_{2}} e^{\beta(t-\tau) L_{3}} & (\tau \leq t \leq T) .\end{cases}
\end{aligned}
$$
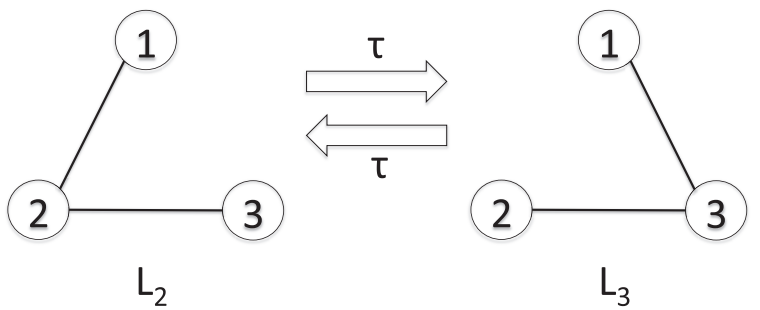

Fig. 1. Network topology of the periodic temporal network in the example. The network structure switches between two topologies described by the Laplacian matrices $L_{2}$ and $L_{3}$ every $\tau$.

We obtain the expansions of $U(t ; 0), S(t)$, and $S^{-1}(t)$ from Eqs. (23), (28), and (29) as

$$
U(t ; 0)= \begin{cases}1-\beta t L_{2}+\frac{1}{2} \beta^{2} t^{2} L_{2}^{2}+O\left(\beta^{3}\right) & (0 \leq t \leq \tau), \\ 1-\beta\left[(t-\tau) L_{3}+\tau L_{2}\right]+\frac{1}{2} \beta^{2}\left[\tau^{2} L_{2}^{2}+2(t-\tau) \tau L_{3} L_{2}+(t-\tau)^{2} L_{3}^{2}\right]+O\left(\beta^{3}\right) & (\tau \leq t \leq T),\end{cases}
$$

and 


$$
\begin{aligned}
S(t)= & \left\{\begin{array}{cc}
1+\frac{1}{2} \beta t\left(L_{3}-L_{2}\right)+\frac{1}{8} \beta^{2} t^{2}\left[L_{2}^{2}-2 L_{2} L_{3}+L_{3}^{2}\right]+O\left(\beta^{3}\right) \\
1+\beta\left(\tau-\frac{t}{2}\right)\left(L_{3}-L_{2}\right)+\frac{1}{2} \beta^{2}\left[\left(\tau-\frac{t}{2}\right)^{2} L_{2}^{2}-t\left(\tau-\frac{t}{2}\right) L_{2} L_{3}\right. \\
\left.+2(t-\tau)\left(\tau-\frac{t}{2}\right) L_{3} L_{2}+\left(\tau-\frac{t}{2}\right)^{2} L_{3}^{2}\right]+O\left(\beta^{3}\right) & (\tau \leq t \leq \tau), \\
S^{-1}(t)=\left\{\begin{array}{cc}
1-\frac{1}{2} \beta t\left(L_{3}-L_{2}\right)+\frac{1}{8} \beta^{2} t^{2}\left[L_{2}^{2}-2 L_{3} L_{2}+L_{3}^{2}\right]+O\left(\beta^{3}\right), \\
1-\beta\left(\tau-\frac{t}{2}\right)\left(L_{3}-L_{2}\right)+\frac{1}{2} \beta^{2}\left\{\left(\tau-\frac{t}{2}\right)^{2} L_{2}^{2}-t\left(\tau-\frac{t}{2}\right) L_{3} L_{2}\right. \\
\left.+2(t-\tau)\left(\tau-\frac{t}{2}\right) L_{2} L_{3}+\left(\tau-\frac{t}{2}\right)^{2} L_{3}^{2}\right\}+O\left(\beta^{3}\right)
\end{array}\right. & (\tau \leq t \leq T),
\end{array}\right.
\end{aligned}
$$

These expansions can also be obtained by substituting

$$
\begin{aligned}
& \int_{0}^{t} d t_{1} L\left(t_{1}\right)= \begin{cases}L_{2} t & (0 \leq t \leq \tau) \\
L_{2} \tau+L_{3}(t-\tau) & (\tau \leq t \leq 2 \tau)\end{cases} \\
& \Lambda_{1}=\frac{1}{T} \int_{0}^{T} d t_{1} L\left(t_{1}\right)=\frac{1}{2}\left(L_{2}+L_{3}\right) \\
& t \int_{0}^{t} d t_{1} L\left(t_{1}\right) \Lambda_{1}= \begin{cases}\frac{1}{2} t^{2}\left(L_{2}^{2}+L_{2} L_{3}\right) & (0 \leq t \leq \tau), \\
\frac{1}{2} t\left[\tau\left(L_{2}^{2}+L_{2} L_{3}\right)+(t-\tau)\left(L_{3} L_{2}+L_{3}^{2}\right)\right] & (\tau \leq t \leq T),\end{cases} \\
& t \int_{0}^{t} d t_{1} \Lambda_{1} L\left(t_{1}\right)= \begin{cases}\frac{1}{2} t^{2}\left(L_{2}^{2}+L_{3} L_{2}\right) & (0 \leq t \leq \tau), \\
\frac{1}{2} t\left[\tau\left(L_{2}^{2}+L_{3} L_{2}\right)+(t-\tau)\left(L_{2} L_{3}+L_{3}^{2}\right)\right] & (\tau \leq t \leq T),\end{cases} \\
& \int_{0}^{t} d t_{1} \int_{0}^{t_{1}} d t_{2} L\left(t_{1}\right) L\left(t_{2}\right)= \begin{cases}\frac{1}{2} t^{2} L_{2}^{2} & (0 \leq t \leq \tau), \\
\frac{1}{2} \tau^{2} L_{2}^{2}+(t-\tau) \tau L_{3} L_{2}+\frac{1}{2}(t-\tau)^{2} L_{3}^{2} & (\tau \leq t \leq T),\end{cases} \\
& \int_{0}^{t_{2}} d t_{1} \int_{0}^{t} d t_{2} L\left(t_{1}\right) L\left(t_{2}\right)= \begin{cases}\frac{1}{2} t^{2} L_{2}^{2} & (0 \leq t \leq \tau), \\
\frac{1}{2} \tau^{2} L_{2}^{2}+(t-\tau) \tau L_{2} L_{3}+\frac{1}{2}(t-\tau)^{2} L_{3}^{2} & (\tau \leq t \leq T),\end{cases}
\end{aligned}
$$

and

$$
\begin{aligned}
\Lambda_{2} & =\frac{1}{T^{2}} \int_{0}^{T} d t_{1} \int_{0}^{t_{1}} d t_{2} L\left(t_{1}\right) L\left(t_{2}\right)=\frac{1}{8}\left(L_{2}^{2}+2 L_{3} L_{2}+L_{3}^{2}\right) \\
t^{2}\left(\Lambda_{1}^{2}-\Lambda_{2}\right) & =\frac{1}{8} t^{2}\left(L_{2}^{2}+2 L_{2} L_{3}+L_{3}^{2}\right)
\end{aligned}
$$

into Eqs. (13) and (14). Therefore, equivalence between the expansion of the exact solution to the second order and our formula has been verified in this particular example. This expansion works even when we do not know the exact solutions of the evolution equation.

\section{Summary and discussion}

In this paper, we studied the series expansion of the matrix characterizing the time evolution of temporal networks which is periodic in time. The essence is to take the lower order terms of the time ordered exponential, and would be applicable in the case of weak coupling of the network. In the future, it is important to apply the proposed method to the analysis and control of the real networks. This formalism may be extended to systems where each element in the network has its own internal dynamics, e.g. synchronization in networks of chaotic oscillators.

The present method works for switching networks as shown in the example. This method can also be applied to hybrid dynamical systems $[23,25]$, where the governing equation switches depending on the dynamical states, if its solution settles down to a periodic state. Application to these systems would be another important problem in the future.

Since the proposed method is based on the series expansion, it is valid when the coupling strength $\beta$ is small enough and higher order terms are negligible. The lowest order expansion corresponds to the simple time average, and the deviation from the time average appears in the second order. Formally, this expansion is possible to be proceeded to an arbitrary order of $\beta$. However, for small enough $\beta$, the lower order terms are enough to approximate the dynamics.

Finally, we discuss the limitation of this method. Since this method is based on the series expansion, it is obvious that this approximation does not work if the coupling is strong so that higher order terms in $\beta$ cannot be neglected. 


\section{Acknowledgments}

This research was supported by JST, CREST.

\section{References}

[1] P. Holme and J. Saramäki, "Temporal networks," Physics Reports, vol. 519, no. 3, pp. 97-125, October 2012.

[2] P. Holme and J. Saramäki (Editors), Temporal networks, Springer, New York, 2013.

[3] T. Takaguchi, M. Nakamura, N. Sato, K. Yano, and N. Masuda, "Predictability of conversation partners," Physical Review X, vol. 1, no. 1, 011008, September 2011.

[4] A. Barrat, M. Barthelemy, and A. Vespignani, Dynamical processes on complex networks, Cambridge University Press, Cambridge, 2008.

[5] N. Fujiwara and J. Kurths, "Spectral universality of phase synchronization in non-identical oscillator networks," European Physical Journal B, vol. 69, no. 1, pp. 45-49, May 2009.

[6] R.M. Anderson and R.M. May, Infectious diseases of humans, Oxford university press, Oxford, 1991.

[7] A. Pikovsky, M. Rosenblum, and J. Kurths, Synchronization: A Universal Concept in Nonlinear Systems, Cambridge University Press, Cambridge, 2003.

[8] Y. Kuramoto, Chemical oscillations, waves, and turbulence, Springer, Berlin; NewYork, 1984.

[9] C. Castellano, S. Fortunato, and V. Loreto, "Statistical physics of social dynamics," Reviews of modern physics, vol. 81, no. 2, pp. 591-646, May 2009.

[10] N. Masuda and P. Holme, "Predicting and controlling infectious disease epidemics using temporal networks," F1000prime reports, vol. 5, no. 6, March 2013.

[11] K. Uriu, Y. Morishita, and Y. Iwasa, "Random cell movement promotes synchronization of the segmentation clock," Proceedings of the National Academy of Sciences of the United States of America, vol. 107, no. 11, pp. 4979-4984, March 2010.

[12] R. Nishi and N. Masuda, "Dynamics of social balance under temporal interaction," EPL (Europhysics Letters), vol. 107, no. 4, 48003, August 2014.

[13] I.V. Belykh, V.N. Belykh, and M. Hasler, "Blinking model and synchronization in small-world networks with a time-varying coupling," Physica D: Nonlinear Phenomena, vol. 195, no. 1, pp. 188-206, August 2004.

[14] M. Frasca, A. Buscarino, A. Rizzo, L. Fortuna, and S. Boccaletti, "Synchronization of moving chaotic agents," Physical Review Letters, vol. 100, no. 4, 044102, January 2008.

[15] M. Porfiri, D.J. Stilwell, E.M. Bollt, and J.D. Skufca, "Random talk: Random walk and synchronizability in a moving neighborhood network," Physica D: Nonlinear Phenomena, vol. 224, no. 1-2, pp. 102-113, December 2006.

[16] D.J. Stilwell, E.M. Bollt, and D.G. Roberson, "Sufficient conditions for fast switching synchronization in time-varying network topologies," SIAM Journal on Applied Dynamical Systems, vol. 5, no. 1, pp. 140-156, August 2006.

[17] N. Fujiwara, J. Kurths, and A. Díaz-Guilera, "Synchronization in networks of mobile oscillators," Physical Review E, vol. 83, no. 2, 025101(R), February 2011.

[18] N. Fujiwara, J. Kurths, and A. Díaz-Guilera, "Spectral analysis of synchronization in mobile networks," AIP Conference Proceedings, vol. 1389, pp. 1015-1018, September 2011.

[19] N. Masuda, K. Klemm, and V.M. Eguíluz, "Temporal networks: Slowing down diffusion by long lasting interactions," Physical Review Letters, vol. 111, no. 18, 188701, October 2013.

[20] J. Zhao, D.J. Hill, and T. Liu, "Synchronization of complex dynamical networks with switching topology: A switched system point of view," Automatica, vol. 45, no. 11, pp. 2502-2511, November 2009.

[21] J. Zhao, D.J. Hill, and T. Liu, "Synchronization of dynamical networks with nonidentical nodes: Criteria and control," IEEE Transactions on Circuits and Systems I: Regular Papers, vol. 58, no. 3, pp. 584-594, March 2011. 
[22] T. Liu, D.J. Hill, and J. Zhao, "Synchronization of dynamical networks by network control," IEEE Transactions on Automatic Control, vol. 57, no. 6, pp. 1574-1580, June 2012.

[23] R.A. Decarlo, M.S. Branicky, S. Pettersson, and B. Lennartson, "Perspectives and results on the stability and stabilizability of hybrid systems," Proceedings of the IEEE, vol. 88, no. 7, pp. 1069-1082, July 2000.

[24] V. Blondel and J.N. Tsitsiklis, "NP-Hardness of some linear control design problems," SIAM Journal on Control and Optimization, vol. 35, no. 6, pp. 2118-2127, November 1997.

[25] R. Goebel, R.G. Sanfelice, and A. Teel, "Hybrid dynamical systems," IEEE Control Systems, vol. 29, no. 2, pp. 28-93, April 2009. 